\title{
Detecting analogical resemblance without retrieving the source analogy
}

\author{
Bogdan Kostic, Anne M. Cleary, Kaye Severin, and Samuel W. Miller \\ Colorado State University, Fort Collins, Colorado
}

\begin{abstract}
We examined whether people can detect analogical resemblance to an earlier experimental episode without being able to recall the experimental source of the analogical resemblance. We used four-word analogies (e.g., robin-nest/beaver-dam), in a variation of the recognition-without-cued-recall method (Cleary, 2004). Participants studied word pairs (e.g., robin-nest) and were shown new word pairs at test, half of which analogically related to studied word pairs (e.g., beaver-dam) and half of which did not. For each test pair, participants first attempted to recall an analogically similar pair from the study list. Then, regardless of whether successful recall occurred, participants were prompted to rate the familiarity of the test pair, which was said to indicate the likelihood that a pair that was analogically similar to the test pair had been studied. Across three experiments, participants demonstrated an ability to detect analogical resemblance without recalling the source analogy. Findings are discussed in terms of their potential relevance to the study of analogical reasoning and insight, as well as to the study of familiarity and recognition memory.
\end{abstract}

Analogical reasoning is a cornerstone of human insight and understanding. Perhaps the best-known empirical demonstration of the role of analogy in insight can be found in Gick and Holyoak's (1980) study of Duncker's (1945) radiation problem. In the radiation problem, participants are informed that a patient has an inoperable, life-threatening stomach tumor. Radiation at levels sufficient to destroy the tumor would also destroy surrounding healthy tissue; lower radiation levels, safe for healthy tissue, would be insufficient to destroy the tumor. The solution is to use many weak rays of radiation, each coming from a different angle, so that the weak rays converge on the tumor. Participants are more likely to reach this solution if they are given an analogically similar problem beforehand, such as the attackdispersion problem, which involves an army attacking a castle via separate roads that converge on the castle.

However, as Gentner, Rattermann, and Forbus (1993, p. 525) noted, "people often fail to access structurally appropriate materials, even when such materials are present in long-term memory." Without having their attention drawn to the potential analogy in memory, only $30 \%$ of participants in Gick and Holyoak's (1980) study reached the solution after being given the analogous problem beforehand. Although many studies have aimed to understand the memory representations and processes involved in analogical reasoning (e.g., Blanchette \& Dunbar, 2000; Green, Fugelsang, \& Dunbar, 2006; Spellman, Holyoak, \& Morrison, 2001; Wharton, Holyoak, \& Lange, 1996), none have examined whether a novel situation that analogically maps onto a situation stored in memory can produce detection of the analogical resemblance when recall of the source analogy fails.
Dual-process theorists of recognition memory argue that two processes contribute to recognition memory: recollection and familiarity (see Yonelinas, 2002, for a review). Recollection-based recognition occurs when one recognizes by bringing to mind the prior episode in question. Familiarity-based recognition occurs when recognition is based on more of a gut feeling that the current situation has been experienced before.

From this dual-process perspective, it is unclear whether analogical resemblance to a prior episode should trigger familiarity. Some researchers have suggested that, whereas familiarity is driven primarily by item-specific properties, recollection is driven by relational processing (e.g., Aggleton \& Brown, 1999; Cleary \& Greene, 2001). Others have suggested that unitization of word pairs at encoding may increase the usefulness of familiarity in making relational/ associative discriminations at test (Quamme, Yonelinas, $\&$ Norman, 2007) and that existing relationships between words at study may encourage unitization (Rhodes \& Donaldson, 2007). Recently, Day and Goldstone (2009) demonstrated that analogical transfer can occur in the absence of explicit awareness of the fact that the new situation maps analogically onto a prior experimental situation. It is possible that participants in their paradigm can experience a general feeling of familiarity with the new, analogically similar situation in the absence of being able to identify why it seems familiar.

The present study investigated the hypothesis that analogical resemblance to a prior episode can be detected in the absence of recalling the source analogy. We used a variation of the recognition-without-cued-recall paradigm (Cleary, 2004): Participants received a recognition test in which half

B. Kostic, bogdan.kostic@colostate.edu 
of the test items resembled a study item on some dimension (e.g., orthography) and half did not. Participants attempted cued recall and rated the likelihood that a word resembling the cue had been studied. Recognition-without-cued-recall is the finding that, among cues that did not elicit successful cued recall, participants discriminated between cues that resembled studied words and cues that did not. This finding suggests that participants detected the increased familiarity with a test cue without retrieving the specific studied item responsible for that increased familiarity.

In the present variation of the recognition-withoutcued-recall paradigm, we used four-word analogies (Green et al., 2006; Spellman et al., 2001). At study, participants were presented with word pairs having preexperimental relationships (e.g., robin-nest). At test, participants received new pairs of words, half of which mapped analogically onto studied pairs (e.g., beaver-dam) and half of which did not (e.g., grape-raisin). For each test pair, participants first attempted to recall a pair from the study list that mapped analogically onto the test pair. Participants then rated the familiarity of the test pair. Our interest was in whether the familiarity ratings would discriminate between pairs that analogically resembled studied pairs and pairs that did not when participants failed to successfully recall the source analogies.

\section{EXPERIMENT 1}

Prior research suggests that participants often do not detect analogical relationships without strong hints (e.g., Gick \& Holyoak, 1980; Spellman et al., 2001). Therefore, Experiment 1 compared a situation in which the relationship between the words was stated explicitly at study with one in which it was not.

\section{Method}

Participants. One hundred two Colorado State University undergraduates participated for course credit: 52 participated in the relationship-stated condition, and 50 participated in the relationshipunstated condition.

Materials. The stimuli were 120 four-word analogies (240 word pairs total). Each word pair (e.g., robin-nest) shared an analogical relationship with one other word pair (e.g., beaver-dam). We created some stimuli and adapted and modified others from Spellman et al. (2001) and from standardized test-preparation materials. We attempted to make each analogical relationship as distinct as possible, such that its two sets of word pairs fit the relationship better than did any other word pairs in the experiment. The semantic overlap between words in corresponding word pairs was also minimized; the only shared attribute between two corresponding sets of word pairs was the relationship between them. For example, we did not use word pairs such as cat-kitten and dog-puppy, because cat could be used to cue recall for $d o g$. See Table 1 for examples of stimuli used in each experiment. The stimuli were counterbalanced so that each word pair appeared equally often at study and at test. ${ }^{1}$

Procedure. The procedure was modified from Cleary (2004). Participants completed four study-test blocks on a computer. In each block, participants viewed a study list of 15 word pairs, followed by a recognition test containing 30 new word pairs, half of which mapped analogically onto studied pairs and half of which did not. In the relationship-stated condition, the relationship between the word pairs was stated explicitly at study, but not at test. As an example of what to expect at study, participants were shown "gas-car, Relationship: fuels." They were then shown coal-train as an example of an analogically related word pair that might appear at test. In the relationship-unstated condition, the relationship was never explicitly stated. Participants were given the same example, but without the statement of the relationship. Participants in both conditions were told to attend to the relationships between words at study, because some word pairs at test might have the same relationship.

Word pairs were randomly assigned to studied and unstudied conditions for each participant. Study items appeared for $3 \mathrm{sec}$ each, with a $1-\mathrm{sec}$ interstimulus interval. Test lists immediately followed the study lists. For each word pair presented at test, participants were first prompted to attempt to recall a word pair from the study list that mapped analogically onto that pair. They were then prompted to provide a familiarity rating between 0 (definitely not studied) and 10 (definitely studied), indicating how familiar the test pair seemed - that is, the likelihood that the test word pair mapped analogically onto a word pair from the study list. After the rating was given, the next test word pair appeared.

Data scoring. Our primary interest was in ratings given when recall of the studied word pair failed. If a participant produced the target word pair for a test trial, the response was considered to be identified. When participants successfully recalled only one word from the analogically similar pair or typed close semantic associates to the pair, these were marked as partially identified. Full and partial identifications were excluded from analyses. Data from 8 partici-

Table 1

Examples of Analogically Related Word Pairs Occurring at Study and Test in Each Experiment

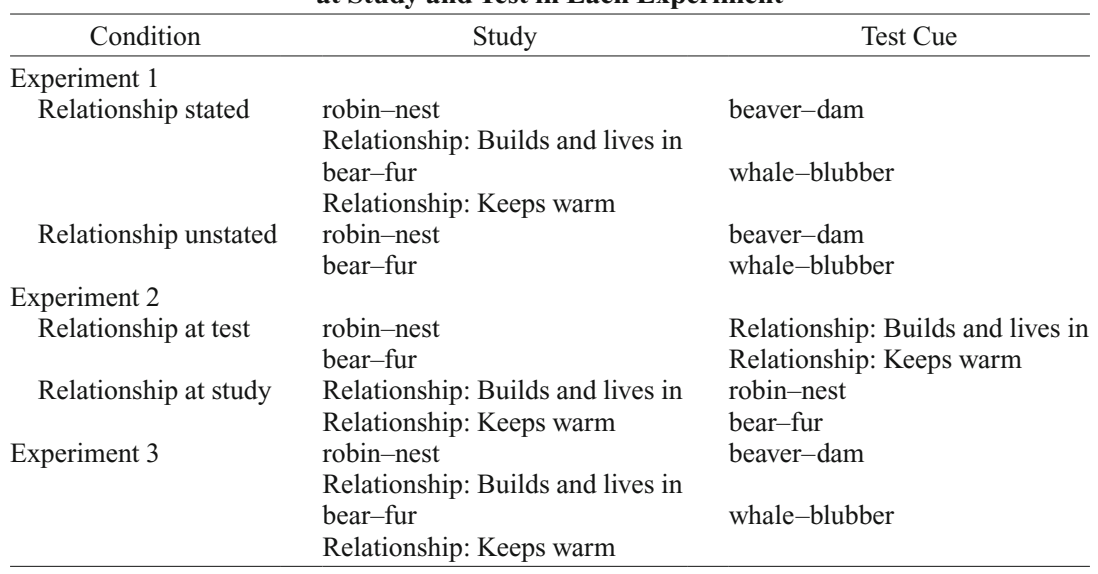


Table 2

Proportion of Analogically Similar Word Pairs Fully and Partially Recalled (Identified) at Test

\begin{tabular}{|c|c|c|c|c|c|c|c|c|}
\hline & \multicolumn{4}{|c|}{ Full Identification Only } & \multicolumn{4}{|c|}{$\begin{array}{l}\text { Full and Partial } \\
\text { Identification }\end{array}$} \\
\hline & \multicolumn{2}{|c|}{ Studied } & \multicolumn{2}{|c|}{ Unstudied } & \multicolumn{2}{|c|}{ Studied } & \multicolumn{2}{|c|}{ Unstudied } \\
\hline & $M$ & $\overline{S D}$ & $M$ & $S D$ & $M$ & $\overline{S D}$ & $M$ & $S D$ \\
\hline \multicolumn{9}{|l|}{ Experiment 1} \\
\hline Relationship stated & .27 & .10 & $<.01$ & $<.01$ & .30 & .11 & $<.01$ & $<.01$ \\
\hline Relationship unstated & .22 & .09 & $<.01$ & .01 & .23 & .10 & $<.01$ & .02 \\
\hline \multicolumn{9}{|l|}{ Experiment 2} \\
\hline Relationship at test & .24 & .10 & $<.01$ & $<.01$ & .25 & .10 & $<.01$ & .01 \\
\hline Relationship at study & .23 & .10 & .09 & .06 & .34 & .12 & .20 & .11 \\
\hline Experiment 3 & .20 & .12 & $<.01$ & $<.01$ & .23 & .13 & $<.01$ & .02 \\
\hline
\end{tabular}

Note- "Full identification" refers to successful identification of the entire analogically similar pair (e.g., typing "robin-nest" in response to beaver-dam); "partial identification" refers to successful identification of only one word from the pair or of words highly similar to the targets (e.g., typing "cops" instead of "police")

pants from the relationship-stated condition were excluded because of a failure to follow directions and for counterbalancing purposes.

\section{Results and Discussion}

Identification rates are presented in Table $2 .{ }^{2}$ The data of primary interest, mean familiarity ratings given on trials without full or partial identification, are presented in Figure 1. Among unidentified test pairs, ratings were higher for test pairs corresponding to studied items in both the relationship-stated $[t(43)=3.80, S E=0.10, p<.001$, Cohen's $d=0.24]$ and the relationship-unstated $[t(49)=$ $3.36, S E=0.07, p<.01, d=0.16$ ] conditions. ${ }^{3}$ Figure 2 shows mean familiarity ratings given to test items for which no identification response was attempted. Here, the effect was still significant in both the relationship-stated $[t(43)=$ 4.64, $S E=0.09, p<.001, d=0.24]$ and the relationshipunstated $[t(49)=2.47, S E=0.08, p<.05, d=0.12]$ conditions. Thus, even when participants were unable to recall the source of the analogical resemblance, they were able to discriminate between test pairs that mapped analogically onto studied pairs and test pairs that did not. ${ }^{4}$ Hereafter, we refer to this effect as the analogical resemblance effect.

The analogical resemblance effect occurred even when the relationships were not stated explicitly at study. To examine whether stating the relationships at study enhanced the effect, we performed a 2 (study status: studied, unstudied) $\times 2$ (condition: relationship stated, relationship unstated) mixed-factor ANOVA on the ratings. The magnitude of the effect was not significantly greater when the relationships were stated explicitly $[F(1,93)=1.41$, $\left.M S_{\mathrm{e}}=0.17, p=.24\right]$.

Examining ratings for unidentified items across blocks may indicate how expectations and practice influenced performance. A 2 (study status: analogy pair studied vs. unstudied) $\times 4$ (block: $1,2,3$, or 4 ) repeated measures ANOVA revealed a main effect of study status in both the relationship-stated $\left[F(1,43)=16.64, M S_{\mathrm{e}}=0.82, p<\right.$ $\left..001, \eta_{\mathrm{p}}^{2}=.28\right]$ and the relationship-unstated $[F(1,49)=$ $\left.11.91, M S_{\mathrm{e}}=0.51, p<.001, \eta_{\mathrm{p}}^{2}=.20\right]$ conditions. There was no interaction in either condition $\left(F_{\mathrm{S}}<1.0\right)$, indicating

Ratings for Unidentified Test Items

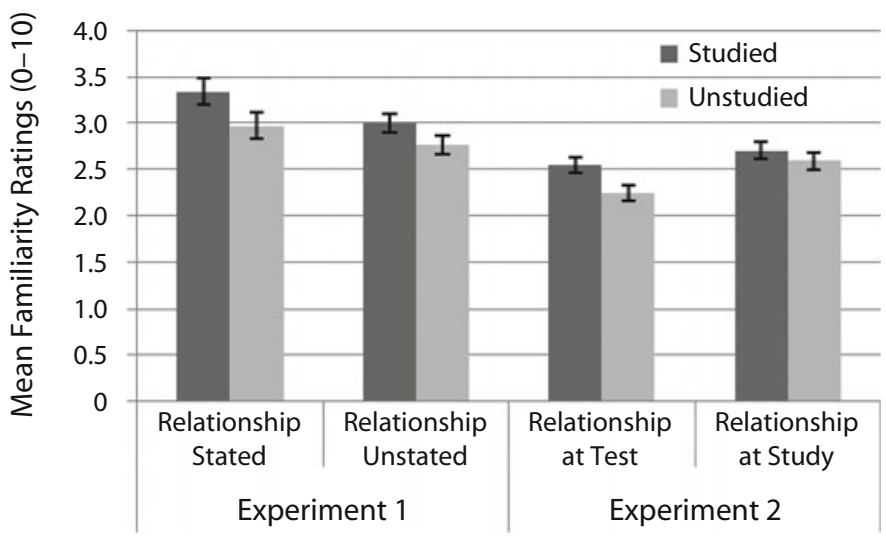

Figure 1. Mean familiarity ratings given to test items for which the study item was unidentified (neither partial nor full identification). Ratings are possible from 0 to 10 . Error bars represent within-subjects confidence intervals (Loftus \& Masson, 1994). 


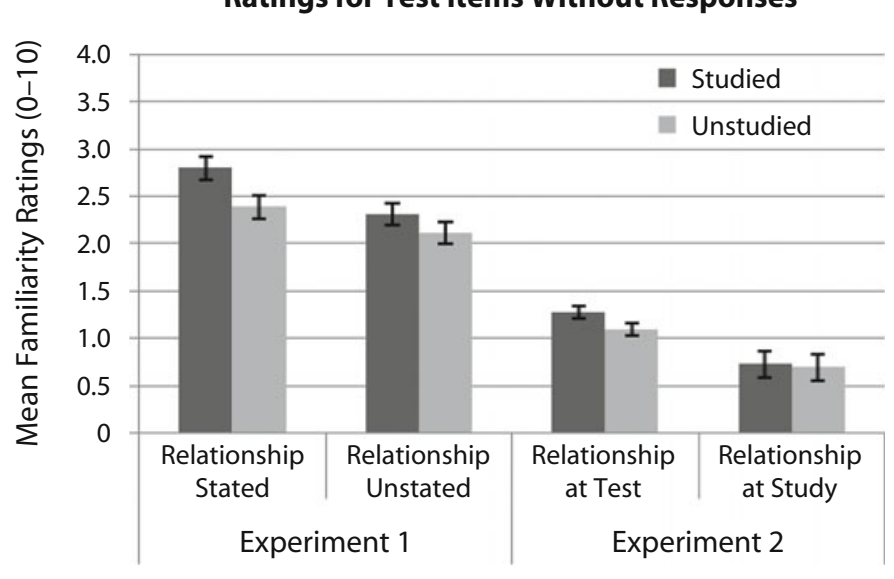

Figure 2. Mean familiarity ratings given to test items for which no response was attempted. Ratings are possible from 0 to 10. Error bars represent within-subjects confidence intervals (Loftus \& Masson, 1994).

that the level of analogically similar/dissimilar discrimination did not differ significantly across blocks. However, a main effect of block emerged, whereby overall ratings tended to increase with block in both the relationshipstated $\left[F(3,129)=10.87, M S_{\mathrm{e}}=1.96, p<.001, \eta_{\mathrm{p}}^{2}=\right.$ $.20]$ and the relationship-unstated $[F(3,147)=4.01$, $\left.M S_{\mathrm{e}}=1.22, p<.01, \eta_{\mathrm{p}}^{2}=.08\right]$ conditions. Participants became less conservative with their familiarity ratings as the experiment progressed, which has been shown in other studies as well (Cleary, Ryals, \& Nomi, 2009). The same overall pattern emerged for trials without responses and in the other experiments reported here. Therefore, the effects of block are not discussed further.

\section{EXPERIMENT 2}

A theoretical question concerning four-word analogies is whether the effects are driven by category comembership of the word pairs or by representations of the relationships between pair members. Some research suggests that processing of four-word analogies can involve an abstraction of the relationship between words in the pairs (e.g., Green et al., 2006). Experiment 2 examined whether this would be the case for the analogical resemblance effect, and if so, whether presenting the word pairs at study is necessary, or if it suffices to present only a statement of their relationship at study. We compared a condition in which only word pairs were studied with one in which only a statement of the relationship was studied. In the former condition, only a statement of the relationship appeared as a cue at test; in the latter condition, only a word pair appeared as a cue at test.

\section{Method}

Participants. One hundred thirty-two Colorado State University undergraduates participated for course credit.

Materials and Procedure. The materials and procedure were identical to those in Experiment 1, with the following exceptions. Participants were randomly assigned to one of two conditions. In the relationships-at-test condition, only word pairs appeared at study and only relationship statements appeared as cues for recalling word pairs at test. In the relationships-at-study condition, only relationship statements appeared at study and only word pairs appeared as cues for recalling relationships at test.

Data scoring. Scoring was the same as in Experiment 1. Data from 6 participants were excluded because of a failure to follow instructions and for counterbalancing purposes.

\section{Results and Discussion}

Mean identification rates are presented in Table 2. Mean familiarity ratings are presented in Figure 1. Familiarity ratings discriminated between studied and unstudied items in the relationships-at-test condition; ratings were higher for relationships corresponding to unidentified studied pairs $[t(61)=5.04, S E=0.06, p<.001, d=0.20]$, even among trials without responses $[t(61)=4.04, S E=0.04$, $p<.001, d=0.12]$. This finding suggests that, at study, some representation of the relationship between words within a pair is encoded; this representation may increase familiarity for relationship statements, even when the studied word pairs themselves are not retrieved. However, the words themselves have to appear at encoding in order for the effect to occur. The analogical resemblance effect was not significant in the relationships-at-study condition $[t(61)=1.59, S E=0.07, p=.12]$, even when only trials without responses were considered $[t(57)=0.26, S E=$ $0.10, p=.80]$.

\section{EXPERIMENT 3}

Although it appears that representations of the relationships between words are involved in the analogical resemblance effect, actually presenting a statement of the relationship at study is neither required nor sufficient for participants' ability to detect analogical resemblance without recalling the source analogy. Still, it is possible that participants articulate the relationship in response to each word pair at study and then recall the relationship itself in response to the corresponding test pair, despite being unable to recall the studied word pair itself. Experiment 3 examined the extent to which participants do this, and whether 
the ability to do so is required for the effect to occur. Toward this end, the relationship was stated, along with each word pair at study, as was the case in Experiment 1; but at test, we asked participants to attempt to identify both the analogically related word pair and the relationship.

\section{Method}

Participants. Ninety-two Colorado State University undergraduates participated for course credit.

Materials and Procedure. The materials and procedure were identical to those in Experiment 1, except that, after being prompted to recall the word pair from study that corresponded to the test pair, participants were prompted to identify the relationship between the words before then being prompted to rate the familiarity of the test pair.

Data scoring. Scoring was the same as in Experiment 1, except that, among the test trials for which identification (or partial identification) of the corresponding word pair did not occur, the trials were further separated into three categories: those for which identification of the exact relationship stated at study occurred; those for which the exact relationship stated at study was unidentified, but the typed relationship could conceivably map between the studied word pair and the test pair; and those for which no analogically mappable relationship between the study and test pairs was identified.

\section{Results and Discussion}

Among test pairs whose corresponding analogically similar word pairs were unidentified, participants were more likely to identify the relationship intended by the experimenters when the pair and their stated relationship was studied $(M=.12, S D=.07)$ than when they were unstudied $[M=.06, S D=.04, t(91)=8.28, S E=0.01, p<.001$, $d=1.05]$. The same was not true when participants identified relationships that differed from what the experimenters had intended, but that could conceivably be mapped between the study and test pairs (e.g., when the participant typed "eats" instead of the intended relationship, "catch with," for the four-word analogy mouse-cheese/fishworm). There was a slightly greater tendency to make such identifications in the unstudied $(M=.32, S D=.17)$ than in the studied $(M=.30, S D=.16)$ condition $[t(91)=1.99$,
$S E=0.01, p=.05, d=0.62]$. This likely reflects the fact that studied pairs had appeared with a statement of the experimenter-intended relationship, leading participants to be more likely to identify the intended relationship in the studied condition.

Among test pairs whose corresponding analogically similar word pairs were unidentified, participants expressed increased familiarity with those whose corresponding word pairs were studied, relative to those whose corresponding word pairs were not studied, in all comparisons (see Table 3). This analogical resemblance effect occurred when participants did not identify the relationship intended by the experimenter - that is, the relationship stated along with the pair at study $[t(91)=3.32$, $S E=0.08, p=.001, d=0.16]$. The effect also occurred even when participants failed to identify any relationship that could conceivably map between the pairs in question $[t(91)=1.96, S E=0.09, p=.05, d=0.11]$. As Table 3 shows, when participants failed to identify the intended relationship but did identify a relationship that could conceivably map between the pairs (not all participants did this), ratings were higher overall than when they could not $\left[F(1,85)=147.73, M S_{\mathrm{e}}=1.77, p<.001, \eta_{\mathrm{p}}^{2}=.64\right]$, but the analogical resemblance effect was still present $[t(85)=$ $3.80, S E=0.10, p<.001, d=0.23]$. The magnitude of the analogical resemblance effect itself did not differ significantly between these two situations, because the interaction was not significant $\left[F(1,85)=2.62, M S_{\mathrm{e}}=0.38, p=\right.$ .11]. Overall, this pattern of results suggests that an ability to recall the relationship from the study list is not required for the analogical resemblance effect to occur.

\section{GENERAL DISCUSSION}

We report three experiments showing participants' detection of analogical resemblance without recall of the source analogy. Experiment 1 showed a variation of the recognition-without-cued-recall effect (Cleary, 2004),

Table 3

Mean Familiarity Ratings Given to Test Word Pairs for Which the Analogy Pair Was Unidentified in Experiment 3

\begin{tabular}{|c|c|c|c|c|}
\hline \multirow[b]{3}{*}{ Identification Results } & \multicolumn{4}{|c|}{ Analogy Pair } \\
\hline & \multicolumn{2}{|c|}{ Studied } & \multicolumn{2}{|c|}{ Unstudied } \\
\hline & $M$ & $S D$ & $M$ & $S D$ \\
\hline Intended relationship unidentified (overall) & 2.81 & 1.54 & 2.56 & 1.53 \\
\hline Different but mappable relationship identified & 4.35 & 1.68 & 3.95 & 1.75 \\
\hline No mappable relationship identified & 2.42 & 1.50 & 2.25 & 1.51 \\
\hline
\end{tabular}

Note- "Intended relationship unidentified (overall)" refers to ratings given when neither the words from the analogy pair nor the specific intended relationship (i.e., that which was stated at study) could be identified at test (nor was there partial recollection of the words). "Different but mappable relationship identified" refers to instances where participants did not identify the intended relationship but thought of a relationship that could conceivably map between the source analogy pair and the target pair (e.g., if the participant typed "eats" as the relationship for fish-worm, whose corresponding pair was mouse-cheese, but the intended relationship was "catch with"). "No mappable relationship identified" refers to instances in which participants either gave a relationship that did not map between the two pairs (e.g., the source analogy pair was windshield-passengers and the participant typed "you wear them" in response to the test pair goggles-eyes) or did not even attempt to guess at a relationship. 
using four-word analogies (e.g., Green et al., 2006; Spellman et al., 2001). Without recalling source analogies (e.g., robin-nest), participants can discriminate between test word pairs that analogically map onto studied pairs (e.g., beaver-dam) and test word pairs that do not. This ability does not depend on presenting a statement of the relationship with each pair at study. This may seem surprising, given that participants often need to have their attention drawn to the analogical relationships in order for many effects to occur (e.g., Spellman et al., 2001); in the present study, however, even when the relationship itself was not stated explicitly, participants were still instructed to attend to the relationship between the words within each pair.

That the analogical resemblance effect occurred when only a statement of the relationship was presented as the retrieval cue at test suggests that it can result from a representation of the analogical relationship (separate from the individual words that composed the analogy). However, although it is likely that a representation of the relationship is involved in the effect, it has to be obtained from the word pair itself and not from just a statement of the relationship, because the effect was absent when participants were given only a statement of the relationship at study and the word pair as a retrieval cue for recalling the relationship from study. Interestingly, participants did not need to be able to articulate the relationship, and did not even need to articulate a mappable relationship between the two pairs in the analogy, to detect analogical resemblance in the absence of recalling the source analogy (Experiment 3).

What drives the analogical resemblance effect reported here? One possibility is that this type of discrimination reflects feelings of familiarity brought on by the resemblance at test. It has long been known that resemblance of a novel situation to a prior experience can trigger feelings of familiarity (e.g., Cleary, 2004). If so, it is possible that participants are aware of a general increased sense of familiarity with test cues that analogically map onto studied items without being aware of the experimental source of that familiarity. Participants may simply have a gut feeling about the test pair without being able to articulate why. The level of awareness may be similar to that occurring in such subjective phenomena as tip-of-the-tongue states or déjà vu experiences (e.g., Cleary \& Reyes, 2009).

An alternative possibility is that the detection of analogical resemblance occurs outside of participants' awareness. Voss and Paller (2009) reported evidence for an unconscious contribution to performance on recognition memory tasks and argued that this contribution differs from familiarity, which, they contend, is explicit. Furthermore, Schunn and Dunbar (1996) and Day and Goldstone (2009) showed that analogical transfer can occur in the absence of explicit awareness of the mapping between the two situations. Specifically, participants can be primed to better solve a problem if it has been preceded by a similar problem, even if they are unaware of the specific link between the two. It is unclear whether participants in these paradigms could have been aware of a general sense of familiarity with the new situation without being able to identify why it seemed familiar. Still, taken together, the results of Voss and Paller, Schunn and Dunbar, and Day and Goldstone suggest that it is at least possible that participants' detection of analogical resemblance in the present study was an unconscious form of memory. Future research may aim to determine the extent to which the detection of analogical resemblance without recall of the source analogy involves explicit familiarity, as opposed to an unconscious, implicit form of memory, and to determine whether such memory may contribute to analogical transfer and analogical problem solving.

\section{AUTHOR NOTE}

This project was supported by National Science Foundation Grant BCS-0638486 to A.M.C. Address correspondence concerning this article to B. Kostic, Department of Psychology, Colorado State University, 1876 Campus Delivery, Fort Collins, CO 80523-1876 (e-mail: bogdan .kostic@colostate.edu).

\section{REFERENCES}

Aggleton, J. P., \& Brown, M. W. (1999). Episodic memory, amnesia, and the hippocampal-anterior thalamic axis. Behavioral \& Brain Sciences, 22, 425-444.

Blanchette, I., \& Dunbar, K. (2000). How analogies are generated: The roles of structural and superficial similarity. Memory \& Cognition, 28, 108-124.

Cleary, A. M. (2004). Orthography, phonology, and meaning: Word features that give rise to feelings of familiarity in recognition. Psychonomic Bulletin \& Review, 11, 446-451.

Cleary, A. M., \& Greene, R. L. (2001). Memory for unidentified items: Evidence for the use of letter information in familiarity processes. Memory \& Cognition, 29, 540-545.

Cleary, A. M., \& Reyes, N. L. (2009). Scene recognition without identification. Acta Psychologica, 131, 53-62.

Cleary, A. M., Ryals, A. J., \& Nomi, J. S. (2009). Can déjà vu result from similarity to a prior experience? Support for the similarity hypothesis of déjà vu. Psychonomic Bulletin \& Review, 16, 1082-1088.

Day, S. B., \& Goldstone, R. L. (2009). Analogical transfer from interaction with a simulated physical system. In Proceedings of the 31 st Annual Conference of the Cognitive Science Society (pp. 1406-1411). Amsterdam: Cognitive Science Society.

DunCKer, K. (1945). On problem solving. Psychological Monographs, 58(5, Whole No. 270).

Dunlap, W. P., Cortina, J. M., Vaslow, J. B., \& Burke, M. J. (1996). Meta-analysis of experiments with matched groups or repeated measures designs. Psychological Methods, 1, 170-177.

Gentner, D., Rattermann, M. J., \& Forbus, K. D. (1993). The roles of similarity in transfer: Separating retrievability from inferential soundness. Cognitive Psychology, 25, 524-575.

Gick, M. L., \& Holyoak, K. J. (1980). Analogical problem solving. Cognitive Psychology, 12, 306-355.

Green, A. E., Fugelsang, J. A., \& Dunbar, K. N. (2006). Automatic activation of categorical and abstract analogical relations in analogical reasoning. Memory \& Cognition, 34, 1414-1421.

Loftus, G. R., \& Masson, M. E. J. (1994). Using confidence intervals in within-subjects designs. Psychonomic Bulletin \& Review, 1, 476-490.

Quamme, J. R., Yonelinas, A. P., \& Norman, K. A. (2007). Effect of unitization on associative recognition in amnesia. Hippocampus, 17, 192-200.

Rhodes, S. M., \& Donaldson, D. I. (2007). Electrophysiological evidence for the influence of unitization on the processes engaged during episodic retrieval: Enhancing familiarity based remembering. Neuropsychologia , 45, 412-424. doi:10.1016/j.neuropsychologia .2006.06.022

Schunn, C. D., \& Dunbar, K. (1996). Priming, analogy, and awareness in complex reasoning. Memory \& Cognition, 24, 271-284.

Spellman, B. A., HolyoaK, K. J., \& Morrison, R. G. (2001). Analogical priming via semantic relations. Memory \& Cognition, 29, 383-393.

Voss, J. L., \& PALler, K. A. (2009). An electrophysiological signature of unconscious recognition memory. Nature Neuroscience, 12, 349-355.

Wharton, C. M., Holyoak, K. J., \& Lange, T. E. (1996). Remote analogical reminding. Memory \& Cognition, 24, 629-643. 
Yonelinas, A. P. (2002). The nature of recollection and familiarity: A review of 30 years of research. Journal of Memory \& Language, 46 441-517.

\section{NOTES}

1. These stimuli are available on request.

2. The identification rates for unstudied items reflect the probability of correctly guessing the targets. There were not enough items in this category to allow for a meaningful comparison of ratings given to identified items.

3. Cohen's $d$ was calculated according to Dunlap, Cortina, Vaslow, and Burke (1996). All recognition-without-cued-recall effects reported herein were replicated in items analyses.

4. Some might be concerned that the effect we report is driven by similarities between items in the analogical pairs. For example, in robin-nest/beaver-dam, participants could be recognizing similarities between robin and beaver or between nest and dam, as opposed to the analogical resemblance between corresponding pairs. To test this, we ran an additional 48 participants in a variation of Experiment 1 in which individual words from one analogical pair (e.g., robin) were studied and individual items from the corresponding analogical pair (e.g., beaver) were presented at test to cue recall for the studied items. We ran versions with the first words from each word pair (robin, beaver) as well as the second words from each word pair (nest, dam), counterbalancing whether each were study or test items. No significant effect emerged $[t(47)=0.81, S E=0.10, p=.42]$, suggesting that our effects were not driven by similarities between individual items.

(Manuscript received January 14, 2009; revision accepted for publication December 16, 2009.) 